\title{
An extension of Darbo's fixed point theorem for a class of system of nonlinear integral equations
}

\author{
Amar Deep ${ }^{1}$, Deepmala', Jamal Rezaei Roshan², Kottakkaran Sooppy Nisar ${ }^{3}$ and \\ Thabet Abdeljawad ${ }^{4,5,6^{*}}$ (D)
}

\author{
"Correspondence: \\ tabdeljawad@psu.edu.sa \\ ${ }^{4}$ Department of Mathematics and \\ General Sciences, Prince Sultan \\ University, 11586 Riyadh, Saudi \\ Arabia \\ ${ }^{5}$ Department of Medical Research, \\ China Medical University, 40402, \\ Taichung, Taiwan \\ Full list of author information is \\ available at the end of the article
}

\begin{abstract}
We introduce an extension of Darbo's fixed point theorem via a measure of noncompactness in a Banach space. By using our extension we study the existence of a solution for a system of nonlinear integral equations, which is an extended result of (Aghajani and Haghighi in Novi Sad J. Math. 44(1):59-73, 2014). We give an example to show the specified existence results.
\end{abstract}

MSC: Primary 47H09; secondary 47H10

Keywords: Fixed point theorem; Nonlinear integral equations (NIE); Tripled fixed point; Measure of noncompactness (MNC)

\section{Introduction and preliminaries}

The degree of noncompactness of a set is measured by means of functions called measures of noncompactness. A quantitative characteristic $\alpha(A)$ measuring the degree of noncompactness of a subset $A$ in a metric space was first considered by Kuratowski [25] in 1930 in connection with problems of general topology. In fixed point theory, one of the most important results is due to Darbo [16], who used this measure to generalize both the classical Schauder fixed point principle and (a special variant of) Banach's contraction mapping principle for so-called condensing operators. A condensing operator is a mapping under which the image of any set is in a certain sense more compact than the set itself. Indeed, the condensing operators have properties similar to those of compact ones. There are some other definitions of measures of noncompactness the authors tried to introduce in an axiomatic way. First, it appeared in the paper of Sadovskii [33], but his axiomatics seems to be too general. In 1980, Banas [9] introduced another axiomatic measure of noncompactness, which was very useful in applications. With the establishment of these comprehensive axiomatics, measures of noncompactness are widely applied in fixed point theory and are especially useful in investigations connected with differential equations, integral equations, functional integral equations, operator equations in Banach spaces [21], fractional differential equations, fractional integral equations, and integro-differential equations [15]. Up

(c) The Author(s) 2020. This article is licensed under a Creative Commons Attribution 4.0 International License, which permits use sharing, adaptation, distribution and reproduction in any medium or format, as long as you give appropriate credit to the original author(s) and the source, provide a link to the Creative Commons licence, and indicate if changes were made. The images or other third party material in this article are included in the article's Creative Commons licence, unless indicated otherwise in a credit line to the material. If material is not included in the article's Creative Commons licence and your intended use is not permitted by statutory regulation or exceeds the permitted use, you will need to obtain permission directly from the copyright holder. To view a copy of this licence, visit http://creativecommons.org/licenses/by/4.0/. 
to now, many authors have presented results on the existence of solutions for the mentioned equations with their applications by using measures of noncompactness and other techniques [1-10, 12-14, 17-20, 22-32, 34].

Here we use the methodology of MNC to enlarge the Darbo fixed point theorem [16]. Our goal is extending the results of [3] from two dimensions to three dimensions and the results of [30] on the existence of three-dimensional fixed points and tripled fixed points for a class of operators in a Banach space.

Throughout this study, we use:

- $\digamma$ : a real Banach space;

- $B(z, \sigma)$ : the closed ball with center $z$ and radius $\sigma$;

- con $Y$ : the convex hull of a set $Y$;

- $\operatorname{co} \bar{Z}$ : the closed convex hull of a set $Z$;

- $M_{\digamma}$ : the set of all bounded subsets of $\digamma$;

- $N_{\digamma}$ : the set of all relatively compact subsets of $\digamma$.

Definition $1.1([10])$. A function $\mu: M_{\digamma} \rightarrow[0,+\infty)$ is said to be MNC in $\digamma$ if it satisfies the following conditions:

$\left(A_{1}\right)$ The family ker $\mu=\left\{Z \in M_{\digamma}: \mu(Z)=0\right\} \neq \emptyset$, and ker $\mu \subseteq N_{\digamma}$.

$\left(A_{2}\right)$ If $Z \subseteq Y$, then $\mu(Z) \leq \mu(Y)$.

$\left(A_{3}\right) \mu(\bar{Z})=\mu(Z)$.

$\left(A_{4}\right) \mu(\operatorname{Conv} Z)=\mu(Z)$.

$\left(A_{5}\right) \mu\left(\lambda_{1} Z+\left(1-\lambda_{1}\right) Y\right) \leq \lambda_{1} \mu(Z)+\left(1-\lambda_{1}\right) \mu(Y)$ for $\lambda_{1} \in[0,1]$.

$\left(A_{6}\right)$ If $\digamma_{r} \in M_{\digamma}$ is such that $Z_{r+1} \subset Z_{r}$ for $r=1,2, \ldots$ and $\lim _{r \rightarrow+\infty} \mu\left(Z_{r}\right)=0$, then $Z_{\infty}=$ $\bigcap_{r=1}^{+\infty} Z_{r} \neq \emptyset$.

Theorem 1.1 (Schauder [2]) Let $\Lambda$ be a nonempty bounded closed convex subset of $\digamma$. Then every compact mapping $F: \Lambda \rightarrow \Lambda$ has at least one fixed point.

Theorem 1.2 (Darbo [9]) Let $F: \Lambda \rightarrow \Lambda$ be a continuous mapping, and let $\Lambda$ be a bounded closed convex subset of $\digamma$. Suppose that there exists a constant $K \in[0,1)$ such that $\mu(F(Z)) \leq K \mu(Z)$ for any $Z \subseteq \Lambda$. Then $F$ has a fixed point.

Definition 1.2 ([11]) A point $(x, y, z)$ is called a tripled fixed point of a mapping $F: Z^{3} \rightarrow Z$ if

$$
F(x, y, z)=x, \quad F(y, x, z)=y, \quad F(z, y, x)=z .
$$

Theorem 1.3 ([10]) Let $\mu_{1}, \mu_{2}, \ldots, \mu_{r}$ be MNCs of $\digamma_{1}, \digamma_{2}, \ldots, \digamma_{r}$, respectively. Moreover, assume that $B: \mathbb{R}_{+}^{r} \rightarrow \mathbb{R}_{+}$is convex and that $B\left(x_{1}, x_{2}, \ldots, x_{r}\right)=0$ iff $x_{j}=0$ for $j=1,2, \ldots, r$. Then

$$
\hat{\mu}(Z)=B\left(\mu_{1}\left(Z_{1}\right), \mu_{2}\left(Z_{2}\right), \ldots, \mu_{r}\left(Z_{r}\right)\right)
$$

defines an MNC in $\digamma_{1} \times \digamma_{2} \times \cdots \times \digamma_{r}$. Here $Z_{j}$ denote the natural projections of $Z$ into $\digamma_{j}$ for $j=1,2, \ldots, r$. 
Example 1.1 ([1]) Let $\mu_{1}, \mu_{2}, \mu_{3}$ be MNCs in $\digamma_{1}, \digamma_{2}, \digamma_{3}$, respectively. Moreover, suppose that $B: \mathbb{R}_{+}^{3} \rightarrow \mathbb{R}_{+}$is convex and that $B\left(x_{1}, x_{2}, x_{3}\right)=0$ iff $x_{j}=0$ for $j=1,2,3$ Then

$$
\hat{\mu}(Z)=B\left(\mu_{1}\left(Z_{1}\right), \mu_{2}\left(Z_{2}\right), \mu_{3}\left(Z_{3}\right)\right)
$$

defines an MNC in $\digamma_{1} \times \digamma_{2} \times \digamma_{3}$. Here $Z_{j}$ denote the natural projections of $Z$ into $\digamma_{j}$ for $j=1,2,3$.

Example 1.2 ([1]) Let $\mu$ be an MNC in $\digamma$, and let $B(x, y, z)=\max \{x, y, z\}$ for $(x, y, z) \in \mathbb{R}_{+}^{3}$. Then $B$ is convex, and if $B(x, y, z)=\max \{x, y, z\}=0$ iff $x=y=z=0$, then clearly all the conditions of Theorem 1.3 are satisfied. Therefore $\hat{\mu}(Z)=\max \left(\mu_{1}\left(Z_{1}\right), \mu_{2}\left(Z_{2}\right), \mu_{3}\left(Z_{3}\right)\right)$ is an MNC on $\digamma \times \digamma \times \digamma$. Here $Z_{j}$ denote the natural projections of $Z$ into $\digamma_{j}$ for $j=1,2,3$.

Example 1.3 ([1]) Let $\mu$ be an MNC in $\digamma$, and let $B(x, y, z)=x+y+z$ for $(x, y, z) \in \mathbb{R}_{+}^{3}$. Then $B$ is convex, and if $B(x, y, z)=x+y+z=0$ iff $x=y=z=0$, the clearly all the conditions of Theorem 1.3 are satisfied. Therefore $\left.\hat{\mu}(Z)=\mu_{1}\left(Z_{1}\right)+\mu_{2}\left(Z_{2}\right)+\mu_{3}\left(Z_{3}\right)\right)$ is an MNC on $\digamma \times \digamma \times \digamma$. Here $Z_{j}$ denote the natural projections of $Z$ into $\digamma_{j}$ for $j=1,2,3$.

Lemma 1.4 (Aghajani [2]) Let $\theta: \mathbb{R}^{+} \rightarrow \mathbb{R}^{+}$be a nondecreasing upper semicontinuous function. Then the following two conditions are equivalent:

(i) $\lim _{n \rightarrow+\infty} \theta^{n}(\zeta)=0, \zeta>0$.

(ii) $\theta(\zeta)<\zeta, \zeta>0$

\section{Main results}

First, we denote by $\hat{\varphi}$ the class of functions $\tilde{\varphi}: \mathbb{R}^{+} \times \mathbb{R}^{+} \times \mathbb{R}^{+} \rightarrow \mathbb{R}^{+}$with the following properties:

(i) $\tilde{\varphi}$ is a nondecreasing continuous function on $\mathbb{R}^{+} \times \mathbb{R}^{+} \times \mathbb{R}^{+}$.

(ii) $\lim _{r \rightarrow+\infty} \hat{\theta}^{r}(\zeta)=0, \zeta>0$, where $\hat{\theta}(\zeta)=\tilde{\varphi}(\zeta, \zeta, \zeta)$.

(iii) $\frac{1}{3}\left(\tilde{\varphi}\left(\zeta_{1}, \nu_{1}, \varphi_{1}\right)+\tilde{\varphi}\left(\zeta_{2}, \nu_{2}, \varphi_{2}\right)+\tilde{\varphi}\left(\zeta_{3}, \nu_{3}, \varphi_{3}\right)\right) \leq \tilde{\varphi}\left(\frac{\zeta_{1}+\zeta_{2}+\zeta_{3}}{3}, \frac{\nu_{1}+\nu_{2}+\nu_{3}}{3}, \frac{\varphi_{1}+\varphi_{2}+\varphi_{3}}{3}\right)$

for all $\zeta_{1}, v_{1}, \varphi_{1}, \zeta_{2}, \nu_{2}, \varphi_{2}, \zeta_{3}, \nu_{3}, \varphi_{3} \in \mathbb{R}_{+}$.

Remark 2.1 If $\tilde{\varphi}(\zeta, \zeta, \zeta)$ is nondecreasing and continuous, then $\hat{\theta}(\zeta)$ is also nondecreasing and continuous. Now by Lemma 1.4 the following two statements are equivalent:

(i) $\lim _{r \rightarrow+\infty} \hat{\theta}^{r}(\zeta)=0, \zeta>0$.

(ii) $\hat{\theta}(\zeta)<\zeta, \zeta>0$

Thus $\tilde{\varphi}(\zeta, \zeta, \zeta)<\zeta, \zeta>0$.

For example, the functions $\tilde{\varphi}(\zeta, v, \varphi)=\ln \left(1+\frac{\zeta+v+\varphi}{3}\right)$ and $\tilde{\varphi}(\zeta, v, \varphi)=U_{1} \zeta+U_{2} v+U_{3} \varphi$, where $U_{1}, U_{2}, U_{3} \in \mathbb{R}^{+}$and $U_{1}+U_{2}+U_{3}<1$, belong to $\hat{\varphi}$.

Theorem 2.2 Let $\Lambda$ be a nonempty bounded closed convex subset of $\digamma$, and let $F: \Lambda \times$ $\Lambda \times \Lambda \rightarrow \Lambda \times \Lambda \times \Lambda$ be a continuous function satisfying

$$
\hat{\mu}(F(Z)) \leq \tilde{\varphi}(\hat{\mu}(Z), \hat{\mu}(Z), \hat{\mu}(Z))
$$

for any subset of $Z$ of $\Lambda \times \Lambda \times \Lambda$, where $\hat{\mu}(Z)$ is defined in Example 1.1, and $\tilde{\varphi} \in \hat{\varphi}$. Then $F$ has at least one fixed point in $\Lambda \times \Lambda \times \Lambda$. 
Proof Define the sequence $\left\{\Lambda_{r} \times \Lambda_{r} \times \Lambda_{r}\right\}_{r=1}^{+\infty}$ by induction: $\Lambda_{0} \times \Lambda_{0} \times \Lambda_{0}=\Lambda \times \Lambda \times \Lambda$, and $\Lambda_{r} \times \Lambda_{r} \times \Lambda_{r}=\operatorname{ConvF}\left(\Lambda_{r-1} \times \Lambda_{r-1} \times \Lambda_{r-1}\right)$ for $r=1,2,3, \ldots$.

We have $F\left(\Lambda_{0} \times \Lambda_{0} \times \Lambda_{0}\right)=F(\Lambda \times \Lambda \times \Lambda) \subseteq \Lambda \times \Lambda \times \Lambda=\Lambda_{0} \times \Lambda_{0} \times \Lambda_{0}$, that is, $\Lambda_{1} \times \Lambda_{1} \times \Lambda_{1} \subseteq \Lambda_{0} \times \Lambda_{0} \times \Lambda_{0}$.

Continuing this way, we can show that

$$
\cdots \subseteq \Lambda_{r} \times \Lambda_{r} \times \Lambda_{r} \subseteq \cdots \subseteq \Lambda_{1} \times \Lambda_{1} \times \Lambda_{1} \subseteq \Lambda_{0} \times \Lambda_{0} \times \Lambda_{0} .
$$

If there exists an integer $P>0$ such that $\hat{\mu}\left(\Lambda_{P} \times \Lambda_{P} \times \Lambda_{P}\right)=0$, then $\Lambda_{P} \times \Lambda_{P} \times \Lambda_{P}$ is relatively compact, and since

$$
F\left(\Lambda_{P} \times \Lambda_{P} \times \Lambda_{P}\right) \subseteq \operatorname{Conv} F\left(\Lambda_{P} \times \Lambda_{P} \times \Lambda_{P}\right)=\Lambda_{P+1} \times \Lambda_{P+1} \times \Lambda_{P+1} \subseteq \Lambda_{P} \times \Lambda_{P} \times \Lambda_{P},
$$

by Theorem 1.1 $F$ has a fixed point. Thus $\hat{\mu}\left(\Lambda_{r} \times \Lambda_{r} \times \Lambda_{r}\right)>0$ for all $r \geq 0$. We obtain

$$
\begin{aligned}
\hat{\mu} & \left(\Lambda_{r+1} \times \Lambda_{r+1} \times \Lambda_{r+1}\right) \\
& =\hat{\mu}\left(\operatorname{Conv} F\left(\Lambda_{r} \times \Lambda_{r} \times \Lambda_{r}\right)\right. \\
& =\hat{\mu}\left(F\left(\Lambda_{r} \times \Lambda_{r} \times \Lambda_{r}\right)\right) \\
& \leq \tilde{\varphi}\left(\hat{\mu}\left(\Lambda_{r} \times \Lambda_{r} \times \Lambda_{r}\right), \hat{\mu}\left(\Lambda_{r} \times \Lambda_{r} \times \Lambda_{r}\right), \hat{\mu}\left(\Lambda_{r} \times \Lambda_{r} \times \Lambda_{r}\right)\right) \\
& =\hat{\theta}\left(\hat{\mu}\left(\Lambda_{r} \times \Lambda_{r} \times \Lambda_{r}\right)\right) \\
& =\hat{\theta}\left(\hat{\mu}\left(\operatorname{Conv} F\left(\Lambda_{r-1} \times \Lambda_{r-1} \times \Lambda_{r-1}\right)\right)\right) \\
& =\hat{\theta}\left(\hat{\mu}\left(F\left(\Lambda_{r-1} \times \Lambda_{r-1} \times \Lambda_{r-1}\right)\right)\right) \\
& \leq \hat{\theta}\left(\tilde{\varphi}\left(\hat{\mu}\left(\Lambda_{r-1} \times \Lambda_{r-1} \times \Lambda_{r-1}\right), \hat{\mu}\left(\Lambda_{r-1} \times \Lambda_{r-1} \times \Lambda_{r-1}\right), \hat{\mu}\left(\Lambda_{r-1} \times \Lambda_{r-1} \times \Lambda_{r-1}\right)\right)\right) \\
& =\hat{\theta}\left(\hat{\mu}\left(\Lambda_{r-1} \times \Lambda_{r-1} \times \Lambda_{r-1}\right)\right) \\
& \leq \cdots \leq \hat{\theta}^{r}\left(\hat{\mu}\left(\Lambda_{1} \times \Lambda_{1} \times \Lambda_{1}\right)\right)
\end{aligned}
$$

Therefore $\hat{\mu}\left(\Lambda_{r+1} \times \Lambda_{r+1} \times \Lambda_{r+1}\right) \rightarrow 0$ as $r \rightarrow+\infty$.

Since $\Lambda_{r+1} \times \Lambda_{r+1} \times \Lambda_{r+1} \subseteq \Lambda_{r} \times \Lambda_{r} \times \Lambda_{r}$ for $r=0,1,2, \ldots$, in view of $\left(A_{6}\right)$, the set $\Lambda_{\infty} \times \Lambda_{\infty} \times \Lambda_{\infty}=\bigcap_{r=1}^{+\infty} \Lambda_{r} \times \Lambda_{r} \times \Lambda_{r}$ is a closed convex subset of $\Lambda \times \Lambda \times \Lambda$ invariant under the operator $F$ and belongs to ker $\hat{\mu}$, that is, $F$ maps $\Lambda_{\infty} \times \Lambda_{\infty} \times \Lambda_{\infty}$ into itself and thus by Theorem 1.1 F has at least one fixed point in $\Lambda_{\infty} \times \Lambda_{\infty} \times \Lambda_{\infty}$ and thus in $\Lambda \times \Lambda \times \Lambda$.

Theorem 2.3 Let $\Lambda$ be a nonempty bounded closed convex subset of $\digamma$, let $\hat{\mu}$ be an arbitrary $M N C$, and let $F: \Lambda \times \Lambda \times \Lambda \rightarrow \Lambda$ be a continuous function satisfying

$$
\mu\left(F\left(Z_{1} \times Z_{2} \times Z_{3}\right)\right) \leq \tilde{\varphi}\left(\mu\left(Z_{1}\right), \mu\left(Z_{2}\right), \mu\left(Z_{3}\right)\right)
$$

for all $Z_{1}, Z_{2}, Z_{3} \subseteq \Lambda$, where $\tilde{\varphi} \in \hat{\varphi}$. Then $F$ has a tripled fixed point.

Proof First, $\hat{\mu}(Z)=\mu\left(Z_{1}\right)+\mu\left(Z_{2}\right)+\mu\left(Z_{3}\right)$ is an MNC in $\digamma \times \digamma \times \digamma$, where $Z_{1}, Z_{2}$, and $Z_{3}$ denote the natural projections of $Z \subseteq \Lambda \times \Lambda \times \Lambda$ into $\digamma$. Let $\hat{F}: \Lambda \times \Lambda \times \Lambda \rightarrow \Lambda \times \Lambda \times \Lambda$ 
be the mapping defined as $\hat{F}(x, y, z)=(F(x, y, z), F(y, x, z), F(z, y, x)$ for $(x, y, z) \in \Lambda \times \Lambda \times \Lambda$. Since $F$ is continuous, $\hat{F}$ is also continuous. Then by Theorem 2.2 we have

$$
\begin{aligned}
\hat{\mu} & (\hat{F}(Z)) \\
& \leq \hat{\mu}\left(F\left(Z_{1} \times Z_{2} \times Z_{3}\right), F\left(Z_{2} \times Z_{1} \times Z_{3}\right), F\left(Z_{3} \times Z_{2} \times Z_{1}\right)\right) \\
& =\mu\left(F\left(Z_{1} \times Z_{2} \times Z_{3}\right)+\mu\left(F\left(Z_{2} \times Z_{1} \times Z_{3}\right)+\mu\left(F\left(Z_{3} \times Z_{2} \times Z_{1}\right)\right.\right.\right. \\
& \leq \tilde{\varphi}\left(\mu\left(Z_{1}\right), \mu\left(Z_{2}\right), \mu\left(Z_{3}\right)\right)+\tilde{\varphi}\left(\mu\left(Z_{2}\right), \mu\left(Z_{1}\right), \mu\left(Z_{3}\right)\right)+\tilde{\varphi}\left(\mu\left(Z_{3}\right), \mu\left(Z_{2}\right), \mu\left(Z_{1}\right)\right) \\
& \leq 3 \tilde{\varphi}\left(\frac{\mu\left(Z_{1}\right)+\mu\left(Z_{2}\right)+\mu\left(Z_{3}\right)}{3}, \frac{\mu\left(Z_{2}\right)+\mu\left(Z_{1}\right)+\mu\left(Z_{3}\right)}{3}, \frac{\mu\left(Z_{3}\right)+\mu\left(Z_{2}\right)+\mu\left(Z_{1}\right)}{3}\right) \\
& \leq 3 \tilde{\varphi}\left(\frac{\hat{\mu}(Z)}{3}, \frac{\hat{\mu}(Z)}{3}, \frac{\hat{\mu}(Z)}{3}\right) .
\end{aligned}
$$

Hence

$$
\frac{1}{3} \hat{\mu}(\hat{F}(Z)) \leq \tilde{\varphi}\left(\frac{\hat{\mu}(Z)}{3}, \frac{\hat{\mu}(Z)}{3}, \frac{\hat{\mu}(Z)}{3}\right) .
$$

Putting $\hat{\mu}_{1}=\frac{1}{3} \hat{\mu}$, we have

$$
\hat{\mu}(\hat{F}(Z)) \leq \tilde{\varphi}(\hat{\mu}(Z), \hat{\mu}(Z), \hat{\mu}(Z)) .
$$

Also, $\hat{\mu}_{1}$ is an MNC. So by Theorem $2.2 F$ has a tripled fixed point.

Corollary 2.4 Let $\Lambda$ be a nonempty bounded closed convex subset of $\digamma$, let $\mu$ be an arbitrary MNC, and let $F: \Lambda \times \Lambda \times \Lambda \rightarrow \Lambda$ be a continuous mapping such that for some nonnegative constants $U_{1}, U_{2}, U_{3}$ with $U_{1}+U_{2}+U_{3}<1$,

$$
\mu\left(F\left(Z_{1} \times Z_{2} \times Z_{3}\right) \leq U_{1} \mu\left(Z_{1}\right)+U_{2} \mu\left(Z_{2}\right)+U_{3} \mu\left(Z_{3}\right)\right.
$$

for all $Z_{1}, Z_{2}, Z_{3} \subseteq \Lambda$. Then $F$ has a tripled fixed point.

Proof Setting $\tilde{\varphi}(\zeta, v, \varphi)=U_{1} \zeta+U_{2} v+U_{3} \varphi$ in Theorem 2.3, we obtain the result.

Corollary 2.5 Let $\Lambda$ be a nonempty closed bounded convex subset of $\digamma$, let $\mu$ be an arbitrary $M N C$, and let $F: \Lambda \times \Lambda \times \Lambda \rightarrow \Lambda$ be a continuous mapping such that

$$
\mu\left(F\left(Z_{1} \times Z_{2} \times Z_{3}\right) \leq \ln \left(1+\frac{\mu\left(Z_{1}\right)+\mu\left(Z_{2}\right)+\mu\left(Z_{3}\right)}{3}\right)\right.
$$

for all $Z_{1}, Z_{2}, Z_{3} \subseteq \Lambda$. Then $F$ has at least one tripled fixed point.

Proof Putting $\tilde{\varphi}(\zeta, v, \varphi)=\ln \left(1+\frac{\zeta+v+\varphi}{3}\right)$ in Theorem 2.3, we obtain the result.

\section{Applications}

Recall that $\digamma=B C\left(\mathbb{R}^{+} \times \mathbb{R}^{+} \times \mathbb{R}^{+}\right)$is the Banach space of all real-valued continuous bounded functions defined on $\mathbb{R}^{+} \times \mathbb{R}^{+} \times \mathbb{R}^{+}$with the standard norm

$$
\|x\|=\sup \{|x(\zeta, \nu, \varphi)|: \zeta, \nu, \varphi \geq 0\}
$$


Let $Z$ be a fixed nonempty bounded subset of $\digamma$ and fix $\tilde{\epsilon}>0, G>0$, and $x \in Z$. The $\bmod$ ulus of continuity of $x$ on $[0, G]$ is defined as

$$
\begin{aligned}
\omega^{G}(x, \tilde{\epsilon})= & \sup \{\mid x(\zeta, \nu, \varphi)-x(\bar{\zeta}, \bar{v}, \bar{\varphi}|: \zeta, \nu, \varphi, \bar{\zeta}, \bar{\nu}, \bar{\varphi} \in[0, G],| \zeta-\bar{\zeta} \mid \leq \epsilon \\
& |\nu-\bar{\nu}| \leq \tilde{\epsilon},|\varphi-\bar{\varphi}| \leq \tilde{\epsilon}\}
\end{aligned}
$$

Further, let

$$
\begin{aligned}
& \omega^{G}(Z, \tilde{\epsilon})=\sup \left\{\omega^{G}(x, \tilde{\epsilon}): x \in Z\right\}, \\
& \omega_{0}^{G}(Z)=\lim _{\tilde{\epsilon} \rightarrow 0} \omega^{G}(Z, \tilde{\epsilon}),
\end{aligned}
$$

and

$$
\omega_{0}(Z)=\lim _{G \rightarrow+\infty} \omega_{0}^{G}(Z)
$$

Besides, for three fixed numbers $\zeta, \nu, \varphi \in \mathbb{R}^{+}$, we define rhe function $\ddot{\mu}$ on the family $M_{\digamma}$ as

$$
\mu(Z)=\omega_{0}(Z)+\lim _{\zeta, v, \varphi \rightarrow+\infty} \sup \operatorname{diam} Z(\zeta, v, \varphi),
$$

where $Z(\zeta, \nu, \varphi)=\left\{z(\zeta, \nu, \varphi): \zeta, \nu, \varphi \in \mathbb{R}^{+}\right\}$, and

$$
\operatorname{diam} Z(\zeta, \nu, \varphi)=\sup \{|x(\zeta, \nu, \varphi)-y(\zeta, \nu, \varphi)|: x, y \in Z\}
$$

Finally, we are going to prove the existence results for the system

$$
\begin{aligned}
x(\zeta, v, \varphi) & \\
= & g(\zeta, v, \varphi, x(\zeta, v, \varphi), y(\zeta, v, \varphi), z(\zeta, v, \varphi), \\
& \int_{0}^{d(\varphi)} \int_{0}^{e(v)} \int_{0}^{k(\zeta)} h(\zeta, v, \varphi, \xi, \rho, \psi, x(\xi, \rho, \psi), y(\xi, \rho, \psi), z(\xi, \rho, \psi) d \xi d \rho d \psi), \\
y(\zeta, v, \varphi) & =g(\zeta, v, \varphi, y(\zeta, v, \varphi), x(\zeta, v, \varphi), z(\zeta, v, \varphi), \\
& \int_{0}^{d(\varphi)} \int_{0}^{e(v)} \int_{0}^{k(\zeta)} h(\zeta, v, \varphi, \xi, \rho, \psi, y(\xi, \rho, \psi), x(\xi, \rho, \psi), z(\xi, \rho, \psi) d \xi d \rho d \psi), \\
z(\zeta, v, \varphi) & g(\zeta, v, \varphi, z(\zeta, \nu, \varphi), y(\zeta, v, \varphi), x(\zeta, v, \varphi), \\
& \int_{0}^{d(\varphi)} \int_{0}^{e(v)} \int_{0}^{k(\zeta)} h(\zeta, v, \varphi, \xi, \rho, \psi, z(\xi, \rho, \psi), y(\xi, \rho, \psi), x(\xi, \rho, \psi) d \xi d \rho d \psi),
\end{aligned}
$$

where $\zeta, \nu, \varphi \in \mathbb{R}^{+}$.

Consider the following assumptions:

(i) $d, e, k: \mathbb{R}^{+} \rightarrow \mathbb{R}^{+}$are continuous. 
(ii) $g: \mathbb{R}^{+} \times \mathbb{R}^{+} \times \mathbb{R}^{+} \times \mathbb{R} \times \mathbb{R} \times \mathbb{R} \times \mathbb{R} \rightarrow \mathbb{R}$ is continuous, and there exist a function $\varphi_{3} \in \hat{\varphi}$ and a nondecreasing continuous function $\varphi_{4}: \mathbb{R}^{+} \rightarrow \mathbb{R}$ with $\varphi_{4}(0)=0$ such that

$$
|g(\zeta, v, \varphi, x, y, z, w)-g(\zeta, v, \varphi, \tilde{x}, \tilde{y}, \tilde{z}, \tilde{w})| \leq \varphi_{3}(|x-\tilde{x}|,|y-\tilde{y}|,|z-\tilde{z}|)+\varphi_{4}(|w-\tilde{w}|)
$$

for all $\zeta, \nu, \varphi \geq 0$ and $x, y, z, \tilde{x}, \tilde{y}, \tilde{z} \in \mathbb{R}$.

(iii) $h: \mathbb{R}^{+} \times \mathbb{R}^{+} \times \mathbb{R}^{+} \times \mathbb{R}^{+} \times \mathbb{R}^{+} \times \mathbb{R}^{+} \times \mathbb{R} \times \mathbb{R} \times \mathbb{R} \rightarrow \mathbb{R}$ is a continuous function such that

$$
\begin{aligned}
Q= & \sup \left\{\mid \int_{0}^{d(\varphi)} \int_{0}^{e(v)} \int_{0}^{k(\zeta)} h(\zeta, v, \varphi, \xi, \rho, \psi, x(\xi, \rho, \psi), y(\xi, \rho, \psi),\right. \\
& z(\xi, \rho, \psi)) d \xi d \rho d \psi \mid \\
& \left.: \zeta, \nu, \varphi, \xi, \rho, \psi \in \mathbb{R}^{+}, x, y, z \in \digamma\right\}
\end{aligned}
$$

is finite. Further,

$$
\begin{aligned}
& \lim _{\zeta, v, \varphi \rightarrow+\infty} \int_{0}^{d(\varphi)} \int_{0}^{e(v)} \int_{0}^{k(\zeta)} \mid h(\zeta, v, \varphi, \xi, \rho, \psi, x(\xi, \rho, \psi), y(\xi, \rho, \psi), z(\xi, \rho, \psi)) \\
& -h(\zeta, \nu, \varphi, \xi, \rho, \psi, \tilde{x}(\xi, \rho, \psi), \tilde{y}(\xi, \rho, \psi), \tilde{z}(\xi, \rho, \psi)) \mid d \xi d \rho d \psi=0
\end{aligned}
$$

for all $x, y, z, \tilde{x}, \tilde{y}, \tilde{z} \in \digamma$.

(iv) $\hat{Q}=\sup \left\{|g(\zeta, v, \varphi, 0,0,0,0)|: \zeta, v, \varphi \in \mathbb{R}^{+}\right\}<+\infty$.

(v) There exists a positive solution $\sigma$ of the inequality

$$
\hat{Q}+\varphi_{3}(\hat{r}, \hat{r}, \hat{r})+\varphi_{4}(Q) \leq \hat{r}
$$

Theorem 3.1 Under hypotheses (i)-(v), equation (1) has at least one solution in the space $\digamma \times \digamma \times \digamma$.

Proof Define the operator $F: \digamma \times \digamma \times \digamma \rightarrow \digamma$ by

$$
\begin{aligned}
F(x, y, z)(\zeta, v, \varphi) \\
=g(\zeta, v, \varphi, x(\zeta, v, \varphi), y(\zeta, v, \varphi), z(\zeta, v, \varphi), \\
\quad \int_{0}^{d(\varphi)} \int_{0}^{e(v)} \int_{0}^{k(\zeta)} h(\zeta, v, \varphi, \xi, \rho, \psi, x(\xi, \rho, \psi), y(\xi, \rho, \psi), z(\xi, \rho, \psi) d \xi d \rho d \psi) .
\end{aligned}
$$

Clearly, $\digamma \times \digamma \times \digamma$ is a Banach space with sup norm

$$
\|(x, y, z)\|_{\digamma \times \digamma \times \digamma}\|=\| x\left\|_{\digamma}+\right\| y\left\|_{\digamma}+\right\| z \|_{\digamma},
$$

where $\|x\|_{\digamma}=\sup \{|x(\zeta, v, \varphi)|: \zeta, v, \varphi \geq 0\},\|y\|_{\digamma}=\sup \{|y(\zeta, v, \varphi)|: \zeta, v, \varphi \geq 0\}$, and $\|z\|_{\digamma}=$ $\sup \{|z(\zeta, v, \varphi)|: \zeta, v, \varphi \geq 0\}$ for $x, y, z \in \digamma$. Then the operator $F(x, y, z)(\zeta, v, \varphi)$ is continuous 
at any $(x, y, z) \in \digamma$. Let $\hat{B}_{\sigma}=\left\{x \in \digamma:\|x\|_{\digamma} \leq \sigma\right\}$. Now we have

$$
\begin{aligned}
\mid(F(x, y, z)(\zeta, v, \varphi) \mid & (\mid g(\zeta, v, \varphi, x(\zeta, v, \varphi), y(\zeta, v, \varphi), z(\zeta, v, \varphi), \\
& \int_{0}^{d(\varphi)} \int_{0}^{e(v)} \int_{0}^{k(\zeta)} h(\zeta, v, \varphi, \xi, \rho, \psi, x(\xi, \rho, \psi), \\
& y(\xi, \rho, \psi), z(\xi, \rho, \psi) d \xi d \rho d \psi) \mid) \\
\leq & (\mid g(\zeta, v, \varphi, x(\zeta, v, \varphi), y(\zeta, v, \varphi), z(\zeta, v, \varphi), \\
& \int_{0}^{d(\varphi)} \int_{0}^{e(v)} \int_{0}^{k(\zeta)} h(\zeta, v, \varphi, \xi, \rho, \psi, x(\xi, \rho, \psi), \\
& y(\xi, \rho, \psi), z(\xi, \rho, \psi) d \xi d \rho d \psi)-g(\zeta, v, \varphi, 0,0,0,0) \mid)+|g(\zeta, v, \varphi, 0,0,0,0)| \\
\leq & \varphi_{3}(\|x\|,\|y\|,\|z\|)+\varphi_{4}(Q)+\hat{Q} \\
\leq & \sigma .
\end{aligned}
$$

Hence $F\left(\hat{B}_{\sigma} \times \hat{B}_{\sigma} \times \hat{B}_{\sigma}\right) \subseteq \hat{B}_{\sigma}$, which means that $F$ is well defined.

Now we prove that $F$ is continuous on $\hat{B}_{\sigma} \times \hat{B}_{\sigma} \times \hat{B}_{\sigma}$. Let $(x, y, z),(\tilde{x}, \tilde{y}, \tilde{z}) \in \hat{B}_{\sigma} \times \hat{B}_{\sigma} \times \hat{B}_{\sigma}$ and $\epsilon>0$ with

$$
\|(x, y, z)-(\alpha, \beta, \gamma)\|_{\digamma \times \digamma \times \digamma}<\frac{\tilde{\epsilon}}{3} .
$$

Now we have

$$
\begin{aligned}
& \mid(F(x, y, z)(\zeta, v, \varphi)-F(\alpha, \beta, \gamma)(\zeta, \nu, \varphi) \mid \\
& =\mid g(\zeta, v, \varphi, x(\zeta, v, \varphi), y(\zeta, v, \varphi), z(\zeta, v, \varphi), \\
& \int_{0}^{d(\varphi)} \int_{0}^{e(v)} \int_{0}^{k(\zeta)} h(\zeta, \nu, \varphi, \xi, \rho, \psi, x(\xi, \rho, \psi) \\
& y(\xi, \rho, \psi), z(\xi, \rho, \psi) d \xi d \rho d \psi)-g(\zeta, \nu, \varphi, \alpha(\zeta, \nu, \varphi), \beta(\zeta, \nu, \varphi), \gamma(\zeta, \nu, \varphi), \\
& \int_{0}^{d(\varphi)} \int_{0}^{e(\nu)} \int_{0}^{k(\zeta)} h(\zeta, \nu, \varphi, \xi, \rho, \psi, \alpha(\xi, \rho, \psi), \beta(\xi, \rho, \psi), \gamma(\xi, \rho, \psi) d \xi d \rho d \psi) \\
& \leq \varphi_{3}(|x-\alpha|,|y-\beta|,|z-\gamma|)+\varphi_{4}\left(\mid \int_{0}^{d(\varphi)} \int_{0}^{e(v)} \int_{0}^{k(\zeta)}\{h(\zeta, \nu, \varphi, \xi, \rho, \psi, x(\xi, \rho, \psi),\right. \\
& y(\xi, \rho, \psi), z(\xi, \rho, \psi))-h(\zeta, \nu, \varphi, \xi, \rho, \psi, \alpha(\xi, \rho, \psi), \\
& \beta(\xi, \rho, \psi), \gamma(\xi, \rho, \psi))\} d \xi d \rho d \psi \mid) \\
& \leq \varphi_{3}(\|x-\alpha\|,\|y-\beta\|,\|z-\gamma\|) \\
& +\varphi_{4}\left(\mid \int_{0}^{d(\varphi)} \int_{0}^{e(v)} \int_{0}^{k(\zeta)}\{h(\zeta, \nu, \varphi, \xi, \rho, \psi, x(\xi, \rho, \psi),\right.
\end{aligned}
$$




$$
\begin{aligned}
& y(\xi, \rho, \psi), z(\xi, \rho, \psi))-h(\zeta, \nu, \varphi, \xi, \rho, \psi, \alpha(\xi, \rho, \psi), \\
& \beta(\xi, \rho, \psi), \gamma(\xi, \rho, \psi))\} d \xi d \rho d \psi \mid) .
\end{aligned}
$$

From (ii) and (iii) it follows that there exists $G>0$ such that for $\zeta, \nu, \varphi>G$,

$$
\begin{aligned}
& \varphi_{4}\left(\int_{0}^{d(\zeta)} \int_{0}^{e(v)} \int_{0}^{k(\varphi)} \mid h(\zeta, v, \varphi, \xi, \rho, \psi, x(\xi, \rho, \psi), y(\xi, \rho, \psi), z(\xi, \rho, \psi))\right. \\
& \quad-h(\zeta, \nu, \varphi, \xi, \rho, \psi, \alpha(\xi, \rho, \psi), \beta(\xi, \rho, \psi), \gamma(\xi, \rho, \psi)) \mid d \xi d \rho d \psi) \leq \frac{\tilde{\epsilon}}{2}
\end{aligned}
$$

for all $x, y, z, \alpha, \beta, \gamma \in \digamma$.

Consider two cases.

Case 1: If $\zeta, \nu, \varphi>G$, then

$$
|F(x, y, z)(\zeta, v, \varphi)-F(\alpha, \beta, \gamma)(\zeta, v, \varphi)| \leq \varphi_{3}\left(\frac{\tilde{\epsilon}}{3}, \frac{\tilde{\epsilon}}{3}, \frac{\tilde{\epsilon}}{3}\right)+\frac{\tilde{\epsilon}}{2}<\frac{\tilde{\epsilon}}{2}+\frac{\tilde{\epsilon}}{2}=\tilde{\epsilon}
$$

Case 2: If $\zeta, v, \varphi \in[0, G]$, then

$$
|F(x, y, z)(\zeta, v, \varphi)-F(\alpha, \beta, \gamma)(\zeta, v, \varphi)| \leq \varphi_{3}\left(\frac{\tilde{\epsilon}}{3}, \frac{\tilde{\epsilon}}{3}, \frac{\tilde{\epsilon}}{3}\right)+\varphi_{4}(\hat{d} \hat{e} \hat{k} \omega)<\frac{\tilde{\epsilon}}{3}+\varphi_{4}(\hat{d} \hat{e} \hat{k} \omega)
$$

where

$$
\begin{aligned}
\omega(\tilde{\epsilon})= & \sup \{|h(\zeta, v, \varphi, \xi, \rho, \psi, x, y, z)-h(\zeta, v, \varphi, \xi, \rho, \psi, \alpha, \beta, \gamma)|: \zeta, \nu, \varphi \in[0, G], \\
& \xi \in[0, \hat{d}], \rho \in[0, \hat{e}], \\
& \left.\psi \in[0, \hat{k}], x, y, z, \alpha, \beta, \gamma \in[-\sigma, \sigma],\|(x, y, z)-(\alpha, \beta, \gamma)\|_{\digamma \times \digamma \times \digamma}<\frac{\tilde{\epsilon}}{2} \cdot\right\},
\end{aligned}
$$

and

$$
\begin{aligned}
& \hat{d}=\sup \{d(\varphi): \varphi \in[0, G]\}, \\
& \hat{e}=\sup \{e(\nu): v \in[0, G]\}, \\
& \hat{k}=\sup \{k(\zeta): \zeta \in[0, G]\} .
\end{aligned}
$$

From the continuity of $h$ on $[0, G] \times[0, G] \times[0, G] \times[0, \hat{k}] \times[0, \hat{e}] \times[0, \hat{d}] \times[-\sigma, \sigma] \times$ $[-\sigma, \sigma] \times[-\sigma, \sigma]$ we infer that $\omega(\tilde{\epsilon}) \rightarrow 0$ as $\tilde{\epsilon} \rightarrow 0$, and from the continuity of $\varphi_{4}$ we obtain $\varphi_{4}(\hat{d} \hat{e} \hat{k} \omega) \rightarrow 0$ as $\tilde{\epsilon} \rightarrow 0$.

Clearly, $F$ is a continuous mapping from $\hat{B}_{\sigma} \times \hat{B}_{\sigma} \times \hat{B}_{\sigma}$ into $\hat{B}_{\sigma}$. Fix $G>0$ and $\tilde{\epsilon}>0$. Choose $\zeta_{1}, \zeta_{2}, v_{1}, v_{2}, \varphi_{1}, \varphi_{2} \in[0, G]$ such that $\left|\zeta_{1}-\zeta_{2}\right| \leq \tilde{\epsilon},\left|v_{1}-v_{2}\right| \leq \tilde{\epsilon},\left|\varphi_{1}-\varphi_{2}\right| \leq \tilde{\epsilon}$. Assume that $\zeta_{1} \leq \zeta_{2}, v_{1} \leq v_{2}, \varphi_{1} \leq \varphi_{2}$, and $(x, y, z) \in\left(Z_{1} \times Z_{2} \times Z_{3}\right)$. We get

$$
\begin{aligned}
& \left|F(x, y, z)\left(\zeta_{2}, v_{2}, \varphi_{2}\right)-F(x, y, z)\left(\zeta_{1}, v_{1}, \varphi_{1}\right)\right| \\
& \leq \mid g\left(\zeta_{2}, v_{2}, \varphi_{2}, x\left(\zeta_{2}, v_{2}, \varphi_{2}\right), y\left(\zeta_{2}, v_{2}, \varphi_{2}\right), z\left(\zeta_{2}, v_{2}, \varphi_{2}\right),\right. \\
& \quad \int_{0}^{d\left(\varphi_{2}\right)} \int_{0}^{e\left(v_{2}\right)} \int_{0}^{k\left(\zeta_{2}\right)} h\left(\zeta_{2}, v_{2}, \varphi_{2}, \xi, \rho, \psi, x(\xi, \rho, \psi), y(\xi, \rho, \psi), z(\xi, \rho, \psi) d \xi d \rho d \psi\right)
\end{aligned}
$$




$$
\begin{aligned}
& -g\left(\zeta_{2}, \nu_{2}, \varphi_{2}, x\left(\zeta_{1}, \nu_{1}, \varphi_{1}\right), y\left(\zeta_{1}, \nu_{1}, \varphi_{1}\right), z\left(\zeta_{1}, \nu_{1}, \varphi_{1}\right),\right. \\
& \int_{0}^{d\left(\varphi_{2}\right)} \int_{0}^{e\left(\nu_{2}\right)} \int_{0}^{k\left(\zeta_{2}\right)} h\left(\zeta_{2}, \nu_{2}, \varphi_{2}, \xi, \rho, \psi, x(\xi, \rho, \psi), y(\xi, \rho, \psi), z(\xi, \rho, \psi) d \xi d \rho d \psi\right) \\
& +\mid g\left(\zeta_{2}, v_{2}, \varphi_{2}, x\left(\zeta_{1}, v_{1}, \varphi_{1}\right), y\left(\zeta_{1}, v_{1}, \varphi_{1}\right), z\left(\zeta_{1}, v_{1}, \varphi_{1}\right)\right. \\
& \int_{0}^{d\left(\varphi_{2}\right)} \int_{0}^{e\left(\nu_{2}\right)} \int_{0}^{k\left(\zeta_{2}\right)} h\left(\zeta_{2}, \nu_{2}, \varphi_{2}, \xi, \rho, \psi, x(\xi, \rho, \psi), y(\xi, \rho, \psi), z(\xi, \rho, \psi) d \xi d \rho d \psi\right) \\
& -g\left(\zeta_{1}, v_{1}, \varphi_{1}, x\left(\zeta_{1}, v_{1}, \varphi_{1}\right), y\left(\zeta_{1}, v_{1}, \varphi_{1}\right), z\left(\zeta_{1}, v_{1}, \varphi_{1}\right),\right. \\
& \int_{0}^{d\left(\varphi_{2}\right)} \int_{0}^{e\left(\nu_{2}\right)} \int_{0}^{k\left(\zeta_{2}\right)} h\left(\zeta_{2}, \nu_{2}, \varphi_{2}, \xi, \rho, \psi, x(\xi, \rho, \psi), y(\xi, \rho, \psi), z(\xi, \rho, \psi) d \xi d \rho d \psi\right) \\
& +\mid g\left(\zeta_{1}, v_{1}, \varphi_{1}, x\left(\zeta_{1}, v_{1}, \varphi_{1}\right), y\left(\zeta_{1}, v_{1}, \varphi_{1}\right), z\left(\zeta_{1}, v_{1}, \varphi_{1}\right)\right. \\
& \int_{0}^{d\left(\varphi_{2}\right)} \int_{0}^{e\left(\nu_{2}\right)} \int_{0}^{k\left(\zeta_{2}\right)} h\left(\zeta_{2}, \nu_{2}, \varphi_{2}, \xi, \rho, \psi, x(\xi, \rho, \psi), y(\xi, \rho, \psi), z(\xi, \rho, \psi) d \xi d \rho d \psi\right) \\
& -g\left(\zeta_{1}, v_{1}, \varphi_{1}, x\left(\zeta_{1}, v_{1}, \varphi_{1}\right), y\left(\zeta_{1}, v_{1}, \varphi_{1}\right), z\left(\zeta_{1}, v_{1}, \varphi_{1}\right)\right. \\
& \int_{0}^{d\left(\varphi_{2}\right)} \int_{0}^{e\left(v_{2}\right)} \int_{0}^{k\left(\zeta_{2}\right)} h\left(\zeta_{1}, \nu_{1}, \varphi_{1}, \xi, \rho, \psi, x(\xi, \rho, \psi), y(\xi, \rho, \psi),\right. \\
& z(\xi, \rho, \psi) d \xi d \rho d \psi) d \delta d \phi d \psi) \\
& +\mid g\left(\zeta_{1}, v_{1}, \varphi_{1}, x\left(\zeta_{1}, v_{1}, \varphi_{1}\right), y\left(\zeta_{1}, v_{1}, \varphi_{1}\right), z\left(\zeta_{1}, v_{1}, \varphi_{1}\right)\right. \\
& \int_{0}^{d\left(\varphi_{2}\right)} \int_{0}^{e\left(v_{2}\right)} \int_{0}^{k\left(\zeta_{2}\right)} h\left(\zeta_{1}, v_{1}, \varphi_{1}, \xi, \rho, \psi, x(\xi, \rho, \psi), y(\xi, \rho, \psi), z(\xi, \rho, \psi) d \xi d \rho d \psi\right) \\
& -g\left(\zeta_{1}, v_{1}, \varphi_{1}, x\left(\zeta_{1}, v_{1}, \varphi_{1}\right), y\left(\zeta_{1}, v_{1}, \varphi_{1}\right), z\left(\zeta_{1}, v_{1}, \varphi_{1}\right)\right. \\
& \int_{0}^{d\left(\varphi_{1}\right)} \int_{0}^{e\left(\nu_{1}\right)} \int_{0}^{k\left(\zeta_{1}\right)} h\left(\zeta_{1}, v_{1}, \varphi_{1}, \xi, \rho, \psi, x(\xi, \rho, \psi), y(\xi, \rho, \psi), z(\xi, \rho, \psi) d \xi d \rho d \psi\right) \\
& \leq \varphi_{3}\left(\left|x\left(\zeta_{2}, \nu_{2}, \varphi_{2}\right)-z\left(\zeta_{1}, \nu_{1}, \varphi_{1}\right)\right|,\left|y\left(\zeta_{2}, \nu_{2}, \varphi_{2}\right)-x\left(\zeta_{1}, \nu_{1}, \varphi_{1}\right)\right|,\right. \\
& \left.\left|z\left(\zeta_{2}, v_{2}, \varphi_{2}\right)-y\left(\zeta_{1}, v_{1}, \varphi_{1}\right)\right|\right) \\
& +\omega_{\sigma}^{G}(g, \epsilon) \\
& +\varphi_{4}\left(\mid \int_{0}^{d\left(\varphi_{2}\right)} \int_{0}^{e\left(v_{2}\right)} \int_{0}^{k\left(\zeta_{2}\right)} h\left(\zeta_{2}, \nu_{2}, \varphi_{2}, \xi, \rho, \psi, x(\xi, \rho, \psi), y(\xi, \rho, \psi), z(\xi, \rho, \psi)\right)\right. \\
& \left.-h\left(\zeta_{1}, v_{1}, \varphi_{1}, \xi, \rho, \psi, x(\xi, \rho, \psi), y(\xi, \rho, \psi), z(\xi, \rho, \psi)\right) d \xi d \rho d \psi \mid\right) \\
& +\varphi_{4}\left(\mid \int_{0}^{d\left(\varphi_{2}\right)} \int_{0}^{e\left(\nu_{2}\right)} \int_{0}^{k\left(\zeta_{2}\right)} h\left(\zeta_{1}, \nu_{1}, \varphi_{1}, \xi, \rho, \psi, x(\xi, \rho, \psi), y(\xi, \rho, \psi),\right.\right. \\
& z(\xi, \rho, \psi)) d \xi d \rho d \psi \\
& -\int_{0}^{d\left(\varphi_{1}\right)} \int_{0}^{e\left(\nu_{1}\right)} \int_{0}^{k\left(\zeta_{1}\right)} h\left(\zeta_{1}, \nu_{1}, \varphi_{1}, \xi, \rho, \psi, x(\xi, \rho, \psi), y(\xi, \rho, \psi),\right.
\end{aligned}
$$




$$
\begin{aligned}
& z(\xi, \rho, \psi))) d \xi d \rho d \psi \mid) \\
& \leq \varphi_{3}\left(\omega^{G}(x, \tilde{\epsilon}), \omega^{G}(y, \tilde{\epsilon}), \omega^{G}(z, \tilde{\epsilon})\right)+\omega_{\sigma}^{G}(g, \tilde{\epsilon})+\varphi_{4}\left(\hat{d} \hat{e} \hat{k} \omega_{\sigma}^{G}(h, \tilde{\epsilon})\right) \\
& \quad+\varphi_{4}\left(\mid \int_{0}^{d\left(\varphi_{2}\right)} \int_{0}^{e\left(v_{2}\right)} \int_{0}^{k\left(\zeta_{2}\right)} h\left(\zeta_{1}, \nu_{1}, \varphi_{1}, \xi, \rho, \psi, x(\xi, \rho, \psi), y(\xi, \rho, \psi),\right.\right. \\
& z(\xi, \rho, \psi)) d \xi d \rho d \psi \\
& -\int_{0}^{d\left(\varphi_{1}\right)} \int_{0}^{e\left(v_{1}\right)} \int_{0}^{k\left(\zeta_{1}\right)} h\left(\zeta_{1}, v_{1}, \varphi_{1}, \xi, \rho, \psi, x(\xi, \rho, \psi), y(\xi, \rho, \psi),\right. \\
& z(\xi, \rho, \psi)) d \xi d \rho d \psi \mid)
\end{aligned}
$$

where

$$
\begin{aligned}
& \omega^{G}(x, \tilde{\epsilon})= \sup \left\{\left|x\left(\zeta_{2}, v_{2}, \varphi_{2}\right)-x\left(\zeta_{1}, v_{1}, \varphi_{1}\right)\right|: \zeta_{1}, \zeta_{2}, v_{1}, v_{2}, \varphi_{1}, \varphi_{2} \in[0, G],\left|\zeta_{1}-\zeta_{2}\right| \leq \tilde{\epsilon},\right. \\
&\left.\left|v_{1}-v_{2}\right| \leq \tilde{\epsilon},\left|\varphi_{1}-\varphi_{2}\right| \leq \tilde{\epsilon}\right\}, \\
& \omega^{G}(y, \tilde{\epsilon})= \sup \left\{\left|y\left(\zeta_{2}, v_{2}, \varphi_{2}\right)-y\left(\zeta_{1}, v_{1}, \varphi_{1}\right)\right|: \zeta_{1}, \zeta_{2}, v_{1}, v_{2}, \varphi_{1}, \varphi_{2} \in[0, G],\left|\zeta_{1}-\zeta_{2}\right| \leq \tilde{\epsilon},\right. \\
&\left.\left|v_{1}-v_{2}\right| \leq \tilde{\epsilon},\left|\varphi_{1}-\varphi_{2}\right| \leq \tilde{\epsilon}\right\}, \\
& \omega^{G}(z, \tilde{\epsilon})= \sup \left\{\left|z\left(\zeta_{2}, v_{2}, \varphi_{2}\right)-z\left(\zeta_{1}, v_{1}, \varphi_{1}\right)\right|: \zeta_{1}, \zeta_{2}, v_{1}, v_{2}, \varphi_{1}, \varphi_{2} \in[0, G],\left|\zeta_{1}-\zeta_{2}\right| \leq \tilde{\epsilon},\right. \\
&\left.\left|v_{1}-v_{2}\right| \leq \tilde{\epsilon},\left|\varphi_{1}-\varphi_{2}\right| \leq \tilde{\epsilon}\right\}, \\
& \omega_{\sigma}^{G}(g, \tilde{\epsilon})= \sup \left\{\left|g\left(\zeta_{2}, v_{2}, \varphi_{2}, x, y, z, w\right)-g\left(\zeta_{1}, \nu_{1}, \varphi_{1}, x, y, z, w\right)\right|:\right. \\
& \zeta_{1}, \zeta_{2}, v_{1}, v_{2}, \varphi_{1}, \varphi_{2} \in[0, G], \\
&\left.\left|\zeta_{1}-\zeta_{2}\right| \leq \tilde{\epsilon},\left|v_{1}-v_{2}\right| \leq \tilde{\epsilon},\left|\varphi_{1}-\varphi_{2}\right| \leq \tilde{\epsilon}, x, y, z \in[-\sigma, \sigma], w \in[-L, L]\right\}, \\
& \omega_{\sigma}^{G}(h, \tilde{\epsilon})= \sup \left\{\left|h\left(\zeta_{2}, v_{2}, \varphi_{2}, \xi, \rho, \psi, x, y, z\right)-h\left(\zeta_{1}, v_{1}, \varphi_{1}, \xi, \rho, \psi, x, y, z\right)\right|:\right. \\
& \zeta_{1}, \zeta_{2}, v_{1}, v_{2}, \varphi_{1}, \varphi_{2}, \in[0, G], \\
&\left|\zeta_{1}-\zeta_{2}\right| \leq \tilde{\epsilon},\left|v_{1}-v_{2}\right| \leq \tilde{\epsilon},\left|\varphi_{1}-\varphi_{2}\right| \leq \tilde{\epsilon}, x, y, z \in[-\sigma, \sigma], \xi \in[0, \hat{k}], \\
&\rho \in[0, \hat{e}], \psi \in[0, \hat{d}]\}, \\
& L=\hat{k} \hat{e} \hat{d} \sup \{\mid h(\zeta, v, \varphi, x(\xi, \rho, \psi), y(\xi, \rho, \psi), z(\xi, \rho, \psi) \mid: \zeta, v, \varphi \in[0, G], \\
&\xi \in[0, \hat{k}], \rho \in[0, \hat{e}], \psi \in[0, \hat{d}], x, y, z \in[-\sigma, \sigma]\} .
\end{aligned}
$$

As $h$ and $g$ are uniformly continuous on $[0, G] \times[0, G] \times[0, G] \times[-\sigma, \sigma] \times[-\sigma, \sigma] \times$ $[-\sigma, \sigma] \times[-L, L]$ and $[0, G] \times[0, G] \times[0, G] \times[0, \hat{k}] \times[0, \hat{e}] \times[0, \hat{d}] \times[-\sigma, \sigma] \times[-\sigma, \sigma] \times$ $[-\sigma, \sigma]$, respectively, we infer that $\omega_{\sigma}^{G}(g, \tilde{\epsilon}), \omega_{\sigma}^{G}(h, \tilde{\epsilon})$ as $\tilde{\epsilon} \rightarrow 0$.

Again, from the uniform continuity of $k, e$, and $d$ on $[0, L]$ we get that $k\left(\zeta_{2}\right) \rightarrow k\left(\zeta_{1}\right)$, $e\left(v_{2}\right) \rightarrow e\left(v_{1}\right)$ and $d\left(\varphi_{2}\right) \rightarrow d\left(\varphi_{1}\right)$ as $\tilde{\epsilon} \rightarrow 0$, So,

$$
\begin{aligned}
& \mid \int_{0}^{d\left(\varphi_{2}\right)} \int_{0}^{e\left(v_{2}\right)} \int_{0}^{k\left(\zeta_{2}\right)} h\left(\zeta_{1}, v_{1}, \varphi_{1}, \xi, \rho, \psi, x(\xi, \rho, \psi), y(\xi, \rho, \psi), z(\xi, \rho, \psi)\right) d \xi d \rho d \psi \\
& \quad-\int_{0}^{d\left(\varphi_{1}\right)} \int_{0}^{e\left(v_{1}\right)} \int_{0}^{k\left(\zeta_{1}\right)} h\left(\zeta_{1}, v_{1}, \varphi_{1}, \xi, \rho, \psi, x(\xi, \rho, \psi), y(\xi, \rho, \psi),\right. \\
& \quad z(\xi, \rho, \psi)) d \xi d \rho d \psi \mid \rightarrow 0,
\end{aligned}
$$


which gives

$$
\begin{aligned}
& \varphi_{2}\left(\mid \int_{0}^{d\left(\varphi_{2}\right)} \int_{0}^{e\left(\nu_{2}\right)} \int_{0}^{k\left(\zeta_{2}\right)} h\left(\zeta_{1}, \nu_{1}, \varphi_{1}, \xi, \rho, \psi, x(\xi, \rho, \psi), y(\xi, \rho, \psi), z(\xi, \rho, \psi)\right) d \xi d \rho d \psi\right. \\
& \quad-\int_{0}^{d\left(\varphi_{1}\right)} \int_{0}^{e\left(v_{1}\right)} \int_{0}^{k\left(\zeta_{1}\right)} h\left(\zeta_{1}, v_{1}, \varphi_{1}, \xi, \rho, \psi, x(\xi, \rho, \psi), y(\xi, \rho, \psi),\right. \\
& \quad z(\xi, \rho, \psi)) d \xi d \rho d \psi \mid) \rightarrow 0
\end{aligned}
$$

as $\tilde{\epsilon} \rightarrow 0$. We have

$$
\omega_{0}^{G}\left(F\left(Z_{1} \times Z_{2} \times Z_{3}\right)\right) \leq \varphi_{3}\left(\omega_{0}^{G}\left(Z_{1}\right), \omega_{0}^{G}\left(Z_{2}\right), \omega_{0}^{G}\left(Z_{3}\right)\right) .
$$

Taking $G \rightarrow+\infty$, we get

$$
\omega_{0}\left(F\left(Z_{1} \times Z_{2} \times Z_{3}\right)\right) \leq \varphi_{3}\left(\omega_{0}\left(Z_{1}\right), \omega_{0}\left(Z_{2}\right), \omega_{0}\left(Z_{3}\right)\right) .
$$

For arbitrary $(x, y, z),(\beta, \gamma, \alpha) \in Z_{1} \times Z_{2} \times Z_{3}$ and $\zeta, v, \varphi \in \mathbb{R}_{+}$, we have

$$
\begin{aligned}
&|F(x, y, z)(\zeta, v, \varphi)-F(\alpha, \beta, \gamma)(\zeta, v, \varphi)| \\
& \leq \varphi_{3}(|x(\zeta, v, \varphi)-\alpha(\zeta, v, \varphi)|,|y(\zeta, v, \varphi)-\beta(\zeta, v, \varphi)|,|z(\zeta, v, \varphi)-\gamma(\zeta, \nu, \varphi)|) \\
& \quad+ \varphi_{4}\left(\mid \int_{0}^{d(\varphi)} \int_{0}^{e(v)} \int_{0}^{k(\zeta)}(h(\zeta, v, \varphi, \xi, \rho, \psi, x(\xi, \rho, \psi), y(\xi, \rho, \psi), z(\xi, \rho, \psi)\right. \\
&-h(\zeta, v, \varphi, \xi, \rho, \psi, \alpha(\xi, \rho, \psi), \beta(\xi, \rho, \psi), \gamma(\xi, \rho, \psi)) d \xi d \rho d \psi \mid), \\
& \leq \varphi_{3}\left(\operatorname { d i a m } \left(Z_{1}(\zeta, v, \varphi), \operatorname{diam}\left(Z_{2}(\zeta, v, \varphi), \operatorname{diam}\left(Z_{3}(\zeta, v, \varphi)\right)\right)\right.\right. \\
& \quad+\varphi_{4}\left(\mid \int_{0}^{d(\varphi)} \int_{0}^{e(v)} \int_{0}^{k(\zeta)}(h(\zeta, v, \varphi, \xi, \rho, \psi, x(\xi, \rho, \psi), y(\xi, \rho, \psi), z(\xi, \rho, \psi)\right. \\
&\quad-h(\zeta, v, \varphi, \xi, \rho, \psi, \alpha(\xi, \rho, \psi), \beta(\xi, \rho, \psi), \gamma(\xi, \rho, \psi)) d \delta d \phi d \psi \mid) .
\end{aligned}
$$

Since $(x, y, z),(\alpha, \beta, \gamma)$, and $\zeta, v, \varphi$ are arbitrary, we get

$$
\begin{aligned}
& \operatorname{diam} F\left(Z_{1} \times Z_{2} \times Z_{3}\right)(\zeta, v, \varphi) \\
& \leq \varphi_{3}\left(\operatorname{diam}\left(Z_{1}(\zeta, v, \varphi), \operatorname{diam}\left(Z_{2}(\zeta, v, \varphi)\right), \operatorname{diam}\left(Z_{3}(\zeta, \nu, \varphi)\right)\right)\right. \\
& \quad+ \varphi_{4}\left(\mid \int_{0}^{d(\varphi)} \int_{0}^{e(v)} \int_{0}^{k(\zeta)}(h(\zeta, v, \varphi, \xi, \rho, \psi, x(\xi, \rho, \psi), y(\xi, \rho, \psi), z(\xi, \rho, \psi)\right. \\
&\quad-h(\zeta, v, \varphi, \xi, \rho, \psi, \alpha(\xi, \rho, \psi), \beta(\xi, \rho, \psi), \gamma(\xi, \rho, \psi)) d \xi d \rho d \psi \mid)
\end{aligned}
$$

As $\zeta, \nu, \varphi \rightarrow+\infty$,

$$
\begin{aligned}
& \lim _{\zeta, v, \varphi \rightarrow+\infty} \sup \operatorname{diam} F\left(Z_{1} \times Z_{2} \times Z_{3}\right)(\zeta, v, \varphi) \\
& \leq \varphi_{3}\left(\lim _{\zeta, v, \varphi \rightarrow+\infty} \sup \operatorname{diam}\left(Z_{1}(\zeta, v, \varphi)\right), \lim _{\zeta, v, \varphi \rightarrow+\infty} \sup \operatorname{diam}\left(Z_{2}(\zeta, v, \varphi)\right),\right. \\
& \left.\quad \lim _{\zeta, v, \varphi \rightarrow+\infty} \sup \operatorname{diam}\left(Z_{3}(\zeta, v, \varphi)\right)\right) .
\end{aligned}
$$


From (2) and (3) we have

$$
\begin{aligned}
\omega( & \left.F\left(Z_{1} \times Z_{2} \times Z_{3}\right)\right)+\lim _{\zeta, v, \varphi \rightarrow+\infty} \sup \operatorname{diam} F\left(Z_{1} \times Z_{2} \times Z_{3}\right)(\zeta, v, \varphi) \\
\leq & \varphi_{3}\left(\omega_{0}\left(Z_{1}\right), \omega_{0}\left(Z_{2}\right), \omega_{0}\left(Z_{3}\right)+\varphi_{3}\left(\lim _{\zeta, v, \varphi \rightarrow+\infty} \sup \operatorname{diam}\left(Z_{1}(\zeta, v, \varphi)\right),\right.\right. \\
& \left.\lim _{\zeta, v, \varphi \rightarrow+\infty} \sup \operatorname{diam}\left(Z_{2}(\zeta, v, \varphi)\right), \lim _{\zeta, v, \varphi \rightarrow+\infty} \sup \operatorname{diam}\left(Z_{3}(\zeta, v, \varphi)\right)\right) \\
\leq & 3 \varphi_{3}\left(\frac{\omega_{0}\left(Z_{1}\right)+\lim _{\zeta, v, \varphi \rightarrow+\infty} \sup \operatorname{diam}\left(Z_{1}(\zeta, v, \varphi)\right)}{3}\right. \\
& \frac{\left.\omega_{0}\left(Z_{2}\right)\right)+\lim _{\zeta, v, \varphi \rightarrow+\infty} \sup \operatorname{diam}\left(Z_{2}(\zeta, v, \varphi)\right)}{3} \\
& \left.\frac{\omega_{0}\left(Y_{3}\right)+\lim _{\zeta, v, \varphi \rightarrow+\infty} \sup \operatorname{diam}\left(Z_{3}(\zeta, v, \varphi)\right)}{3}\right) .
\end{aligned}
$$

Therefore

$$
\frac{1}{3} \mu\left(F\left(Z_{1} \times Z_{2} \times Z_{3}\right)\right) \leq \varphi_{3}\left(\frac{\mu}{3}, \frac{\mu}{3}, \frac{\mu}{3}\right) .
$$

Putting $\frac{1}{3} \mu=\hat{\mu}$, we get

$$
\hat{\mu}\left(F\left(Z_{1} \times Z_{2} \times Z_{3}\right)\right) \leq \varphi_{3}\left(\hat{\mu}\left(Z_{1}\right), \hat{\mu}\left(Z_{2}\right), \hat{\mu}\left(Z_{3}\right)\right) .
$$

Hence equation (1) has a tripled fixed point in the space $\digamma \times \digamma \times \digamma$, and thus the system has a solution in $\digamma \times \digamma \times \digamma$.

Example 3.1 Consider the system of NIEs

$$
\begin{aligned}
x(\zeta, v, \varphi) & =\frac{1}{11} e^{-\left(\zeta^{3}+v^{3}+\varphi^{3}\right)}+\frac{\zeta^{3} \ln (1+x(\zeta, v, \varphi))}{5\left(1+\nu^{3}\right)}+\frac{e^{-v^{2}} \ln (1+y(\zeta, v, \varphi)}{9}+\frac{\varphi^{2} \ln (1+z(\zeta, v, \varphi)}{4\left(1+\varphi^{2}\right)} \\
& +\ln \left(1+\frac{1}{3} \int_{0}^{\varphi} \int_{0}^{v} \int_{0}^{\zeta}\left(\cos ^{2}\left(1+2 \xi^{3} x(\xi, \rho, \psi)\right)+\sin \left(\psi^{3} y(\xi, \rho, \psi)\right)\right.\right. \\
& \left.\left.+\cos \left(\rho^{5} z(\xi, \rho, \psi)\right) d \xi d \rho d \psi\right) / e^{\zeta^{2} v^{2} \varphi^{2}}\right) \\
y(\zeta, v, & \\
= & \frac{1}{11} e^{-\left(\zeta^{3}+v^{3}+\varphi^{3}\right)}+\frac{\zeta^{3} \ln (1+y(\zeta, v, \varphi))}{5\left(1+\nu^{3}\right)}+\frac{e^{-v^{2}} \ln (1+x(\zeta, v, \varphi)}{9} \\
& +\frac{\varphi^{2} \ln (1+z(\zeta, v, \varphi)}{4\left(1+\varphi^{2}\right)} \\
& +\ln \left(1+\frac{1}{3} \int_{0}^{\varphi} \int_{0}^{v} \int_{0}^{\zeta}\left(\cos ^{2}\left(1+2 \xi^{3} y(\xi, \rho, \psi)\right)+\sin \left(\psi^{3} x(\xi, \rho, \psi)\right)\right.\right. \\
& \left.\left.+\cos \left(\rho^{5} z(\xi, \rho, \psi)\right) d \xi d \rho d \psi\right) / e^{\zeta^{2} v^{2} \varphi^{2}}\right)
\end{aligned}
$$




$$
\begin{aligned}
& z(\zeta, \nu, \varphi) \\
& =\frac{1}{11} e^{-\left(\zeta^{3}+\nu^{3}+\varphi^{3}\right)}+\frac{\zeta^{3} \ln (1+z(\zeta, \nu, \varphi))}{5\left(1+\nu^{3}\right)}+\frac{e^{-\nu^{2}} \ln (1+y(\zeta, \nu, \varphi)}{9}+\frac{\varphi^{2} \ln (1+x(\zeta, \nu, \varphi)}{4\left(1+\varphi^{2}\right)} \\
& \quad+\ln \left(1+\frac{1}{3} \int_{0}^{\varphi} \int_{0}^{v} \int_{0}^{\zeta}\left(\cos ^{2}\left(1+2 \xi^{3} z(\xi, \rho, \psi)\right)+\sin \left(\psi^{3} y(\xi, \rho, \psi)\right)\right.\right. \\
& \left.\left.\quad+\cos \left(\rho^{5} x(\xi, \rho, \psi)\right) d \xi d \rho d \psi\right) / e^{\zeta^{2} v^{2} \varphi^{2}}\right) .
\end{aligned}
$$

This system is a particular form of (1) with

$$
\begin{aligned}
& g(\zeta, v, \varphi, x, y, z, w)=\frac{1}{11} e^{-\left(\zeta^{3}+\nu^{3}+\varphi^{3}\right)}+\frac{\zeta^{3} \ln (1+x(\zeta, \nu, \varphi))}{5\left(1+\zeta^{3}\right)}+\frac{e^{-\nu^{2}} \ln (1+y(\zeta, \nu, \varphi)}{9} \\
& +\frac{\varphi^{2} \ln (1+z(\zeta, \nu, \varphi)}{4\left(1+\varphi^{2}\right)}+\ln \left(1+\frac{|w|}{3}\right), \\
& =\frac{\cos ^{2}\left(1+2 \delta^{3} x(\xi, \rho, \psi)\right)+\sin \left(\phi^{3} y(\xi, \rho, \psi)\right)+\cos \left(\psi^{5} z(\xi, \rho, \psi)\right) d \xi d \rho d \psi}{e^{\zeta^{2} v^{2} \varphi^{2}}},
\end{aligned}
$$

$\varphi_{3}(\zeta, v, \varphi)=\ln \left(1+\frac{\zeta+v+\varphi}{3}\right), d(\varphi)=\varphi, e(v)=v, k(\zeta)=\zeta, \varphi_{4}(\zeta)=\frac{\zeta}{3}$.

Now we show that all the properties of Theorem 3.1 are satisfied for system (4).

(i) $d, e, k, g$ are continuous, $|g(\zeta, v, \varphi, 0,0,0,0)|=\frac{1}{11} e^{-\left(\zeta^{3}+v^{3}+\varphi^{3}\right)}$ is bounded for $\zeta, v, \varphi \in$ $\mathbb{R}^{+}$, and $\hat{Q}=\frac{1}{11}$.

(ii) Let $\zeta, v, \varphi \in \mathbb{R}^{+}$and $x, y, z, w, \bar{x}, \bar{y}, \bar{z}, \bar{w} \in \mathbb{R}^{+}$with $|x| \geq|\bar{x}|,|y| \geq|\bar{y}|,|z| \geq|\bar{z}|,|w| \geq|\bar{w}|$. By the mean value theorem, for $\left.\ln \left(1+\frac{|w|}{3}\right)\right)$ and $\ln \left(1+\frac{\zeta+\nu+\varphi}{3}\right) \in \hat{\varphi}$, we have

$$
\begin{aligned}
&|g(\zeta, v, \varphi, x, y, z, w)-g(\zeta, v, \varphi, \tilde{x}, \tilde{y}, \tilde{z}, \tilde{w})| \\
& \leq \frac{\zeta^{3}}{5\left(1+\zeta^{3}\right)}|\ln (1+|x|)|-\ln (1+|\tilde{x}|)\left|+\frac{e^{-v^{2}}}{9}\right| \ln (1+|y|)|-\ln (1+|\tilde{y}|)| \\
&+\frac{\varphi^{2}}{4\left(1+\varphi^{2}\right)}|\ln (1+|z|)|-\ln (1+|\tilde{z}|)|+| \ln \left(1+\frac{|w|}{3}\right)-\ln \left(1+\frac{|\tilde{w}|}{3}\right) \mid \\
& \leq \frac{\zeta^{3}}{5\left(1+\zeta^{3}\right)}\left|\ln \left(\frac{1+|x|}{1+|\tilde{x}|}\right)\right|+\frac{e^{-v^{2}}}{9}\left|\ln \left(\frac{1+|y|}{1+|\tilde{y}|}\right)\right| \\
&+\frac{\varphi^{2}}{4\left(1+\varphi^{2}\right)}\left|\ln \left(\frac{1+|z|}{1+|\tilde{z}|}\right)\right|+\frac{1}{3}\left|\ln \left(\frac{1+\frac{|w|}{3}}{1+\frac{|\tilde{w}|}{3}}\right)\right| \\
& \leq \frac{1}{5}\left|\ln \left(\frac{1+|x|}{1+|\tilde{x}|}\right)\right|+\frac{1}{9}\left|\ln \left(\frac{1+|y|}{1+|\tilde{y}|}\right)\right| \\
&+\frac{1}{4}\left|\ln \left(\frac{1+|z|}{1+|\tilde{z}|}\right)\right|+\frac{1}{3}|w-\tilde{w}| \\
& \leq \frac{1}{4}\left|\ln \left(1+\frac{|x|-|\tilde{x}|}{1+|\tilde{x}|}\right)\right|+\frac{1}{4}\left|\ln \left(1+\frac{|y|-|\tilde{y}|}{1+|\tilde{y}|}\right)\right| \\
& \quad+\frac{1}{4}\left|\ln \left(1+\frac{|z|-|\tilde{z}|}{1+|\tilde{z}|}\right)\right|+\frac{1}{3}|w-\tilde{w}|
\end{aligned}
$$




$$
\begin{aligned}
& \leq \frac{1}{4} \ln (1+|x-\tilde{x}|)+\frac{1}{4} \ln (1+|y-\tilde{y}|)+\frac{1}{4} \ln (1+|z-\tilde{z}|)+\frac{1}{3}|w-\tilde{w}| \\
& \leq \ln \left(1+\frac{|x-\tilde{x}|+|y-\tilde{y}|+|z-\tilde{z}|}{3}\right)+\frac{1}{3}|w-\tilde{w}|, \\
& =\varphi_{3}(|x-\tilde{x}|,|y-\tilde{y}|,|z-\tilde{z}|)+\varphi_{4}(|w-\tilde{w}|) .
\end{aligned}
$$

(iii) Since $h$ is continuous, for each $\zeta, v, \varphi, \xi, \rho, \psi \in \mathbb{R}^{+}$and $x, y, z, \tilde{x}, \tilde{y}, \tilde{z} \in \mathbb{R}^{+}$, we have

$$
\begin{aligned}
& \mid h(\zeta, v, \varphi, \xi, \rho, \psi, x(\xi, \rho, \psi), y(\xi, \rho, \psi), z(\xi, \rho, \psi) \\
& \quad-|h(\zeta, v, \varphi, \xi, \rho, \psi, \tilde{x}(\xi, \rho, \psi), \tilde{y}(\xi, \rho, \psi), \tilde{z}(\xi, \rho, \psi))| \\
& \quad \leq \frac{6}{e^{\zeta^{2} \nu^{2} \varphi^{2}}}, \\
& \quad \lim _{\zeta, \nu, \varphi \rightarrow+\infty} \int_{0}^{\varphi} \int_{0}^{v} \int_{0}^{\zeta} \mid h(\zeta, v, \varphi, \xi, \rho, \psi, x(\xi, \rho, \psi), y(\xi, \rho, \psi), z(\xi, \rho, \psi) \\
& \quad-|h(\zeta, v, \varphi, \xi, \rho, \psi, \tilde{x}(\xi, \rho, \psi), \tilde{y}(\xi, \rho, \psi), \tilde{z}(\xi, \rho, \psi))| \leq \lim _{\zeta, \nu, \varphi \rightarrow+\infty} \frac{6 \zeta v \varphi}{e^{\zeta^{2} v^{2} \varphi^{2}}}=0
\end{aligned}
$$

for all $x, y, z, \tilde{x}, \tilde{y}, \tilde{z} \in \digamma$.

Moreover,

$$
|h(\zeta, v, \varphi, \xi, \rho, \psi, x(\xi, \rho, \psi), y(\xi, \rho, \psi), z(\xi, \rho, \psi))| \leq \frac{3}{e^{\zeta^{2} v^{2} \varphi^{2}}} .
$$

Also,

$$
\int_{0}^{\varphi} \int_{0}^{\nu} \int_{0}^{\zeta}|h(\zeta, v, \varphi, \xi, \rho, \psi, x(\xi, \rho, \psi), y(\xi, \rho, \psi), z(\xi, \rho, \psi)) d \xi d \rho d \psi| \leq \frac{3 \zeta v \varphi}{e^{\zeta^{2} \nu^{2} \varphi^{2}}}
$$

for all $\zeta, v, \varphi, \xi, \rho, \psi \in \mathbb{R}^{+}$and $x, y, z \in \mathbb{R}$. Thus

$$
Q=\sup \left\{\frac{3 \zeta \nu \varphi}{e^{\zeta^{2} \nu^{2} \varphi^{2}}}: \zeta, \nu, \varphi \geq 0\right\}=\frac{3}{\sqrt{2 e}}=1.2866
$$

(iv) Putting all values $Q, \hat{Q}, \varphi_{3}$, and $\varphi_{4}$ in the inequality.

$$
\frac{1}{11}+\ln (1+\hat{r})+0.4286<\hat{r}
$$

For $\hat{r} \geq 2$, we obtain

$$
\hat{r}-\frac{1}{11}-\ln (1+\hat{r})-0.4286>0 .
$$

Choosing $\sigma=2$, all the conditions of Theorem 3.1 are satisfied, and the system of NIEs (4) has a solution in the space $\digamma \times \digamma \times \digamma$.

\section{Conclusions}

There are different generalizations of Darbo's fixed point theorem. Some authors have created generalizations via measures of noncompactness. On the other hand, several authors 
have extended Darbo's fixed point theorem by changing the domain of mappings that possess a fixed point. In this paper, we used contractions to verify that a mapping defined on a nonempty convex bounded closed subset of a given Banach space has at least one fixed point. We prove the existence of solutions for a system of functional nonlinear integral equations in three dimensions.

\section{Acknowledgements}

The first author is thankful to the CSIR JRF Fellowship under the Government of India, Program No. 09/1174(0003)/2017-EMR-1, CSIR New Delhi.

\section{Funding}

The author T. Abdeljawad would like to thank Prince Sultan University for funding this work through research group Nonlinear Analysis Methods in Applied Mathematics (NAMAM), group number RG-DES-2017-01-17.

Availability of data and materials

Not applicable.

\section{Competing interests}

The authors declare that they have no competing interests.

\section{Authors' contributions}

The authors contributed equally and significantly in writing this paper. All authors read and approved the final manuscript.

\section{Author details}

'Mathematics Discipline, PDPM-Indian Institute of Information Technology, Design and Manufacturing, Jabalpur, MP, India. ${ }^{2}$ Department of Mathematics, Qaemshahr Branch, Islamic Azad University, Qaemshahr, Iran. ${ }^{3}$ Department of Mathematics, College of Arts and Sciences, Prince Sattam bin Abdulaziz University, 11991 Wadi Aldawaser, Saudi Arabia. ${ }^{4}$ Department of Mathematics and General Sciences, Prince Sultan University, 11586 Riyadh, Saudi Arabia. ${ }^{5}$ Department of Medical Research, China Medical University, 40402, Taichung, Taiwan. ${ }^{6}$ Department of Computer Science and Information Engineering, Asia University, 40402, Taichung, Taiwan.

\section{Publisher's Note}

Springer Nature remains neutral with regard to jurisdictional claims in published maps and institutional affiliations.

Received: 26 May 2020 Accepted: 30 August 2020 Published online: 09 September 2020

\section{References}

1. Aghajani, A., Allahyari, R., Mursaleen, M.: A generalization of Darbo theorem with application to the solvability of system of integral equations. J. Comput. Appl. Math. 260, 68-77 (2014)

2. Aghajani, A., Banas, J., Sabzali, N.: Some generalizations of Darbo fixed point theorem and applications. Bull. Belg. Math. Soc. Simon Stevin 20(2), 345-358 (2013)

3. Aghajani, A., Haghighi, A.S.: Existence of solutions for a system of integral equations via measure of noncompactness. Novi Sad J. Math. 44(1), 59-73 (2014)

4. Ahmed, I., Kumam, P., Jarad, F., Borisut, P., Sitthithakerngkiet, K., Ibrahim, A.: Stability analysis for boundary value problems with generalized nonlocal condition via Hilfer-Katugampola fractional derivative. Adv. Differ. Equ. 2020(1), $1(2020)$

5. Ahmed, I., Kumam, P., Shah, K., Borisut, P., Sitthithakerngkiet, K., Demba, M.A.: Stability results for implicit fractional pantograph differential equations via $\phi$-Hilfer fractional derivative with a nonlocal Riemann-Liouville fractional integral condition. Mathematics 8(1), 94 (2020)

6. Aleksić, S., Kadelburg, Z., Mitrović, Z.D., Radenović, S.: A new survey: cone metric spaces. J. Int. Math. Virtual Inst. 9, 93-121 (2019)

7. Banaei, S.: An extension of Darbo theorem and its application to existence of solution for a system of integral equations. Cogent Math. Stat. 6, Article ID 1614319 (2019)

8. Banaei, S., Mursaleen, M., Parvaneh, V.: Some fixed point theorems via measure of noncompactness with applications to differential equations. Comput. Appl. Math. 39, 139 (2020). https://doi.org/10.1007/s40314-020-01164-0

9. Banas, J.: On measures of noncompactness in Banach space. Comment. Math. Univ. Carol. 21, 131-143 (1980)

10. Banas, J., Goebel, K.: Measures of Noncompactness in Banach Space. Lecture Notes in Pure and Applied Mathematics, vol. 60. Dekker, New York (1980)

11. Berinde, V., Borcut, M.: Tripled fixed point theorems for contractive type mapping in partially ordered metric space. Nonlinear Anal. 74, 4889-4897 (2011)

12. Bonsal, F.F.: Lecture on Some Fixed Point Theorems of Functional Analysis. Tata, Bombay (1962)

13. Borisut, P., Kumam, P., Ahmed, I., Jirakitpuwapat, W.: Existence and uniqueness for $\psi$-Hilfer fractional differential equation with nonlocal multi-point condition. Math. Methods Appl. Sci. https://doi.org/10.1002/mma.6092

14. Borisut, P., Kumam, P., Ahmed, I., Sitthithakerngkiet, K.: Nonlinear Caputo fractional derivative with nonlocal Riemann-Liouville fractional integral condition via fixed point theorems. Symmetry 11(6), 829 (2019)

15. Corduneanu, C.: Integral Equations and Applications. Cambridge University Press, New York (1990) 
16. Darbo, G.: Punti uniti in transformazioni a codomio non compatto. Rend. Semin. Mat. Univ. Padova 24, 84-92 (1955)

17. Das, A., Hazarika, B., Arab, R., Mursaleen, M.: Application of a fixed point theorem to the existence of solutions to the nonlinear functional integral equations in two variables. Rend. Circ. Mat. Palermo 2 Ser. 68, 139-152 (2019)

18. Deep, A., Deepmala, Tunç, C.: On the existence of solutions of some non-linear functional integral equations in Banach algebra with applications. Arab J. Basic Appl. Sci. 27(1), 279-286 (2020)

19. Deepmala, Pathak, H.K.: A study on some problems on existence of solutions for some nonlinear functional-integral equations. Acta Math. Sci. 33B(5), 1305-1313 (2013)

20. Deepmala, Pathak, H.K.: Study on existence of solutions for some nonlinear functional-integral equations with applications. Math. Commun. 18, 97-107 (2013)

21. Deimling, K.: Nonlinear Functional Analysis. Springer, Berlin (1985)

22. Deng, G., Huang, H., Cvetković, M., Radenović, S.: Cone valued measure of noncompactness and related fixed point theorems. Bull. Int. Math. Virtual Inst. 8, 233-243 (2018)

23. Işık, H., Banaei, S., Golkarmanesh, F., Parvaneh, V., Park, C., Khorshidi, M.: On new extensions of Darbo's fixed point theorem with applications. Symmetry 12(3), 424 (2020). https://doi.org/10.3390/sym12030424

24. Koskela, P., Manojlović, V.: Quasi-nearly subharmonic functions and quasiconformal mappings. Potential Anal. 37(2), 187-196 (2012)

25. Kuratowski, K.: Sur les espaces complets. Fundam. Math. 5, 301-309 (1930)

26. Matani, B., Roshan, J.R.: An existence theorem of tripled fixed point for a class of operators on Banach space with applications. Mat. Vesn. 72(1), 17-29 (2020)

27. Matani, B., Roshan, J.R., Hussain, N.: An extension of Darbo's theorem via measure of non-compactness with its application in the solvability of a system of integral equations. Filomat 33(19), 6315-6334 (2019)

28. Nashine, H.K., Kadelburg, Z., Radenović, S.: Coupled common fixed point theorems for $W^{*}$-compatible mappings in ordered cone metric spaces. Appl. Math. Comput. 218(9), 5422-5432 (2012)

29. Nashine, H.K., Roshan, J.R.: Fixed point theorem via measure of noncompactness and application to Volterra integral equations in Banach algebras. J. Math. Ext. 13(4), 91-116 (2019)

30. Nasiri, H., Roshan, J.R.: The solvability of a class of system of nonlinear integral equations via measure of noncompactness. Filomat 32(17), 5969-5991 (2018)

31. Rahimi, H., Radenović, S., Rad, G.S., Kumam, P.: Quadrupled fixed point results in abstract metric spaces. Comput. Appl. Math. 33(3), 671-685 (2014)

32. Roshan, J.R.: Existence of solutions for a class of system of functional integral equation via measure of noncompactness. J. Comput. Appl. Math. 313, 129-141 (2017)

33. Sadovskii, B.N.: Limit compact and condensing operators. Russ. Math. Surv. 27, 86-144 (1972)

34. Todorčević, V.: Harmonic Quasiconformal Mappings and Hyperbolic Type Metrics. Springer, Berlin (2019)

\section{Submit your manuscript to a SpringerOpen ${ }^{\circ}$ journal and benefit from:}

- Convenient online submission

- Rigorous peer review

- Open access: articles freely available online

- High visibility within the field

- Retaining the copyright to your article

Submit your next manuscript at $>$ springeropen.com 